11

\title{
Электромагнитное излучение линейно и нелинейно осциллирующей заряженной капли
}

\author{
(C) А.И. Григорьев, С.О. Ширяева \\ Ярославский государственный университет им. П.Г. Демидова, \\ 150000 Ярославль, Россия \\ e-mail: grig@uniyar.ac.ru
}

(Поступило в Редакцию 9 февраля 2016 г.)

Показано, что в аналитических расчетах интенсивности электромагнитного излучения осциллирующей заряженной капли в линейном по амплитуде осцилляций приближении (малый параметр 0.1) обнаруживается только квадрупольная составляющая полного излучения. Дипольная компонента может быть обнаружена только в расчетах более высоких порядков приближений. Тем не менее интенсивность дипольного излучения оказывается существенно более высокой (на 14-15 порядков). Это связано с тем, что само разделение излучения системы зарядов на мультипольные компоненты (различающиеся уже разными скоростями убывания потенциала с расстоянием) производится разложением по существенно более малому параметру: отношению размеров излучающей системы (в нашем случае капли радиусом $\sim 10 \mu \mathrm{m}$ ) к расстоянию до точки наблюдения в „волновой зоне“ излучения (к длине излучаемой волны) $100-1000 \mathrm{~m}$. В итоге этот второй малый параметр будет иметь порядок $10^{-7}-10^{-8}$. Отношение же интенсивностей квадрупольного и дипольного излучений согласно теории поля, пропорционально квадрату отношения гидродинамической скорости движения осциллирующей поверхности заряженной капли к скорости распространения электромагнитного сигнала в вакууме (скорости света), что дает $10^{-14}-10^{-15}$.

\section{Введение}

Задача об электромагнитном излучении от осциллирующих заряженных облачных и дождевых капель представляет интерес в связи с проблемами радиолокации метеорологических объектов [1-6] (в частности, см. монографии $[1,2]$ и указанную в них литературу). Проблема расчета интенсивности электромагнитного излучения от осциллирующей заряженной сферической капли в линейном по амплитуде осцилляций приближении была впервые сформулирована в [3], там же были приведены первые оценки применительно к конвективным облакам. Правда, в [3] расчет проводился на основе весьма грубой модели, в которой интенсивность излучения оценивалась по степени рассеяния энергии осцилляций капли идеальной жидкости. Идеи [3] в том же линейном приближении были развиты в [4-7]. Но о виде излучения (дипольное оно или квадрупольное) в [3-7] не упоминалось.

В $[8,9]$ проведены аналитические расчеты электромагнитного излучения от осциллирующей заряженной капли уже во втором порядке малости по амплитуде осцилляций. Неожиданно оказалось, что интенсивность излучения в расчетах второго порядка существенно (на много порядков) выше, чем в расчетах линейного приближения (можно сравнить, например, результаты линейных и нелинейных расчетов $[7,9])$.

Из общей теории электромагнитного излучения (см., например, $[10,11]$ ) следует, что общее излучение может быть представлено в виде суммы дипольного, квадрупольного и магнитно-дипольного. Когда дипольный момент системы ускоренно движущихся зарядов равен нулю, самое интенсивное излучение - это квадру- польное. Магнитно-дипольное излучение часто вообе отсутствует (например, когда магнитный момент системы зарядов пропорционален механическому или когда отношение заряда к массе у всех движущихся частиц одинаково). Отношение интенсивностей квадрупольного и дипольного излучений от данной системы зарядов, согласно [11], порядка квадрата отношения скорости движения зарядов системы к скорости распространения электромагнитного сигнала в вакууме. Это как раз и дает ту величину, которая вытекает из сравнения результатов расчетов в $[7,9]$. Нужно понять, почему дипольное излучение появляется в асимптотических расчетах в более высоких порядках малости по амплитуде осцилляций, чем первый, а квадрупольное излучение (если это оно обнаружено в качественных расчетах [3-7]) обнаруживается уже в расчетах первого порядка малости. Анализу этой не стандартной ситуации и посвящена настоящая работа.

\section{Постановка задачи}

Рассмотрим каплю идеально проводящей несжимаемой невязкой жидкости радиуса $R$ с коэффициентом поверхностного натяжения $\gamma$, имеющую температуру $T$ и несущую заряд $Q$. Примем, что окружающая среда вакуум, а сила тяжести отсутствует. В капле реализуется капиллярное волновое движение весьма малой амплитуды, существующее уже в силу теплового движения молекул [12] и приводящее к малому искажению $\xi(\theta, t)$ равновесной сферической формы поверхности капли, причем $\max |\xi| \ll R$. 
Примем для простоты, что возмущенная форма капли имеет осевую симметрию (что осцилляции осесимметричны). В этом случае интересующие нас физические величины можно раскладывать по осесимметричным полиномам Лежандра [13], что упрощает громоздкость рассмотрения, но не снижает общности рассуждений.

Нижеследующее изложение проведем в соответствии с работой [14], посвященной исследованию электростатической устойчивости заряженной сферической капли, выполненной авторами с целью облегчить понимание работы Рэлея [15], в которой было опущено большинство математических выкладов.

Однако в данном рассмотрении будем интересоваться способностью заряженной капли излучать при осцилляциях электромагнитные волны.

При изучении такого объекта, как осесимметрично осциллирующая заряженная капля, можно рассмотреть некий „эффективный“ заряд $Q$, помещенный внутрь капли, электрическое поле которого на больших расстояниях от капли совпадает с электрическим полем заряженной капли. Положение этого „эффективного“ заряда при осцилляциях поверхности капли также будет осциллировать вдоль выделенной оси в силу принятой осевой симметрии осцилляций. Следовательно, этот „эффективный“ заряд будет источником электромагнитных волн, аналогичным осциллирующей заряженной капле. Поскольку „эффективный“ заряд осциллирует вдоль выделенной оси, то генерируемое излучение будет дипольным.

Будем полагать волновое движение жидкости в капле потенциальным и примем, что поле скоростей движения жидкости в капле $\mathbf{V}(\mathbf{r}, t)=\nabla \psi(\mathbf{r}, t)$ полностью определяется функцией потенциала скорости $\psi(\mathbf{r}, t)$.

Оценим положение „эффективного“ заряда, амплитуду его осцилляций, ускорение его движения и интенсивность дипольного излучения.

Математическая формулировка задачи об электромагнитном излучении осциллирующей заряженной капли записывается в виде $[8,9]$

$$
\begin{gathered}
\Delta \psi(\mathbf{r}, t)=0, \quad \operatorname{div} \mathbf{E}(\mathbf{r}, t)=0, \\
r \rightarrow 0: \quad \psi(\mathbf{r}, t) \rightarrow 0 ; \quad r \rightarrow \infty: \quad \mathbf{E}(\mathbf{r}, t) \rightarrow 0 ; \\
r=R+\xi(\theta, t): \\
\frac{\partial \xi(\theta, t)}{\partial t}=\frac{\partial \psi(\mathbf{r}, t)}{\partial r}-\frac{1}{r^{2}} \frac{\partial \xi(\theta, t)}{\partial \theta} \frac{\partial \psi(\mathbf{r}, t)}{\partial \theta} ; \\
\Delta P-\rho \frac{\partial \psi(\mathbf{r}, t)}{\partial t}+\frac{1}{8 \pi}(\mathbf{E}(\mathbf{r}, t))^{2}=\gamma(\nabla,(\mathbf{r}, t)), \\
\nabla \Phi(\mathbf{r}, t)=\Phi_{0} .
\end{gathered}
$$

Кроме того, запишем условия неизменности полного объема капли (следствие несжимаемости жидкости), неподвижности ее центра масс, а также условие сохранения полного заряда капли

$$
\begin{gathered}
\int_{V} r^{2} d r \sin \theta d \theta d \varphi=\frac{4}{3} \pi R^{3}, \quad \int_{V} \mathbf{r} r^{2} d r \sin \theta d \theta d \varphi=0, \\
V=[0 \leq r \leq 1+\xi(\theta, t), \quad 0 \leq \theta \leq \pi, \quad 0 \leq \varphi \leq 2 \pi], \\
-\frac{1}{4 \pi} \oint_{S}(\mathbf{n}, \nabla \Phi) d S=Q, \\
S=[r=1+\xi(\theta, 1), \quad 0 \leq \theta \leq \pi, \quad 0 \leq \varphi \leq 2 \pi] .
\end{gathered}
$$

В выписанных выражениях введены обозначения: $\Delta P$ - перепад постоянных давлений внутри и вне капли в состоянии равновесия, $\mathbf{n}(\mathbf{r}, t)$ - единичный вектор нормали к возмущенной поверхности капли, $\mathbf{E}(\mathbf{r}, t)-$ вектор напряженности электрического поля собственного заряда капли, $\Phi(\mathbf{r}, t)$ - электрический потенциал такой, что $\mathbf{E}(\mathbf{r}, t)=-\nabla \Phi(\mathbf{r}, t), \Phi_{0}-$ постоянный вдоль поверхности капли потенциал.

\section{Расчет поверхностной плотности заряда на возмущенной капле}

Как отмечалось выше, будем придерживаться схемы вывода, использованной в [14]. В этой связи представим уравнение образующей возмущенной поверхности капли $r(\theta, t)=R+\xi(\theta, t)$ в виде

$$
r(\theta, t)=a_{*}+\sum_{n=0}^{\infty} a_{n}(t) P_{n}(\cos \theta),
$$

где $r$ - расстояние от начала координат до точки поверхности; $a_{n} \ll a_{*} \forall n, P_{n}(\cos \theta)$ - полиномы Лежандра [13], $a_{*}$ - постоянный коэффициент, близкий к радиусу капли (отличающийся от радиуса на величину второго порядка малости, см. ниже (3a)).

Учтем также, что в силу условий сохранения объема капли и положения ее центра масс радиальные осцилляции в капле невозможны, как невозможно и поступательное движение [16]. Это означает, что $a_{0} \equiv a_{1} \equiv 0$.

Примем, что отношения $a_{n} / a_{*}$ малы $\left(\left|a_{n} / a_{*}\right| \ll 1\right)$ и убывают с увеличением номера моды $n$ так, что $\left|a_{n} / a_{n+1}\right|<1$.

Вычислим объем капли

$$
\begin{aligned}
V & =\int_{0}^{2 \pi} \int_{0}^{r(\theta, t)} \int_{0}^{\pi} r^{2} \sin \theta d \varphi d r d \theta \\
& =\frac{2}{3} \pi \int_{0}^{\pi} r(\theta, t)^{3} \sin \theta d \theta=\frac{2}{3} \mu \int_{-1}^{1} r(\theta, t)^{3} d \mu,
\end{aligned}
$$

$d V=r^{2} \sin \theta d \theta d \varphi d r, \quad \mu \equiv \cos \theta, \quad d \mu=-\sin \theta d \theta$, 
где $r=r(\mu)$ определяется (3). В итоге, подставив (3) в (4), получим с точностью до квадратичных слагаемых по $a_{n} / a_{*}$ :

$$
\begin{aligned}
V & \approx \frac{2 \pi}{3} \int_{-1}^{1} a_{*}^{3}\left[1+3 \sum_{n=0}^{\infty} \frac{a_{n}}{a_{*}} P_{n}(\mu)+3 \sum_{n=0}^{\infty} \sum_{n=0}^{\infty} \frac{a_{n} a_{m}}{a_{*}^{2}} P_{n} P_{m}\right] d \mu \\
& =\frac{4}{3} \pi a_{*}^{3}\left[1+3 \sum_{n=0}^{\infty} \frac{1}{(2 n+1) !}\left(\frac{a_{n}}{a_{*}}\right)^{2}\right] .
\end{aligned}
$$

С другой стороны, вводя радиус равновесной сферической капли $R$, ее объем можно записать в виде $V=4 \pi R^{3} / 3$. Приравнивая два последних выражения для объема капли, получим выражение для радиуса равновесной сферической капли $R$ через амплитуды капиллярных волн $a_{n}$ :

$$
R \approx a_{8}\left[1+\sum_{n=1}^{\infty}(2 n+1)^{-1}\left(\frac{a_{n}}{a_{*}}\right)^{2}\right] .
$$

Из (5) несложно видеть, что $a_{*}$ отличается от $R$ на величину второго порядка малости по $a_{n} / a_{*}$.

Вычислим распределение электрического заряда по возмущенной осцилляциями поверхности капли. Перепишем (3) с учетом (5) с точностью до малых первого порядка в виде

$$
\begin{aligned}
r & =a_{*}+\sum_{n=1}^{\infty} a_{n}(t) P_{n}(\mu) \approx R+\sum_{n=1}^{\infty} a_{n}(t) P_{n}(\mu) \\
& \equiv R\left[1+\sum_{n=1}^{\infty} \frac{a_{n}(t)}{R} P_{n}(\mu)\right] .
\end{aligned}
$$

При записи (3a) учтено, что $R$ отличается от $a_{*}$ во втором порядке малости.

Электрический потенциал у свободной поверхности проводящей деформированной, согласно (3а), капли, являющийся решением задачи (1), (2), может быть представлен в виде разложения по полиномам Лежандра

$$
\Phi(\theta, t)=\frac{Q}{r}+\sum_{n=1}^{\infty} \frac{B_{n}(t)}{r^{n+1}} P_{n}(\mu),
$$

где $B_{n}(t)$ - зависящие от времени коэффициенты, для $n \geq 1$ выполняется неравенство $\left|B_{n}(t)\right|>\left|B_{n+1}(t)\right|$.

Поверхность проводника является эквипотенциальной. Обозначив ее потенциал через $\Phi_{0}$ и используя (3a), запишем

$$
\begin{aligned}
\Phi_{0}= & \frac{Q}{R+\sum_{n}^{\infty} a_{n}(t) P_{n}(\mu)} \\
& +\sum_{n}^{\infty} \frac{B_{n}(t)}{\left(R+\sum_{m}^{\infty} a_{m}(t) P_{m}(\mu)\right)^{1+n}} P_{n}(\mu) .
\end{aligned}
$$

Раскладывая это выражение по малым величинам $a_{n}(t) / R$ и $B_{n}(t) / R$ и ограничиваясь линейными слагаемыми (пренебрегая произведениями $B_{n}(t) / R$ на $\left.a_{m}(t) / R\right)$, получим

$$
\Phi_{0} \approx \frac{Q}{R}-\frac{Q}{R} \sum_{n=1}^{\infty} \frac{a_{n}(t)}{R} P_{n}(\mu)+\sum_{n=1}^{\infty} \frac{B_{n}(t)}{R^{n+1}} P_{n}(\mu) .
$$

Приравнивая коэффициенты при полиномах одного порядка, стоящие слева и справа от знака равенства, найдем с точностью до малых первого поряка

$$
\Phi_{0} \approx \frac{Q}{R}, \quad B_{n} \approx Q a_{n} R^{n-1} .
$$

В результате выражение для потенциала электрического поля деформированной капли (6) можно переписать в следующем виде:

$$
\Phi(r, \theta, t)=\frac{Q}{r}+\frac{Q}{r} \sum_{n=1}^{\infty} \frac{a_{n}(t)}{r} \frac{R^{n-1}}{r^{n-1}} P_{n}(\mu) .
$$

Чтобы найти значение плотности заряда $\sigma=\sigma(\theta, t)$ в любой точке свободной поверхности капли, воспользуемся формулой

$$
4 \pi \sigma(\theta, t)=-\left.\mathbf{n} \nabla \Phi\right|_{r=R+\Sigma a_{n} P_{n}} \approx-\left.\frac{\partial \Phi}{\partial r}\right|_{r=R+\Sigma a_{n} P_{n}} \cos \vartheta,
$$

где вектор нормали к возмущенной поверхности капли отличается от вектора нормали к невозмущенной поверхности капли на величину второго порядка малости. Так как $\cos \vartheta$ отличается от единицы на малую величину, пропорциональную $a_{n}^{2}$, примем $\cos \vartheta=1$, тогда

$$
\begin{aligned}
4 \pi \sigma(\theta, t) \approx-\left.\frac{\partial \Phi}{\partial r}\right|_{r=R+\Sigma a_{n} P_{n}} \\
=\left[\frac{Q}{r^{2}}+\sum_{n=1}^{\infty}(n+1) Q R^{n-1} r^{-n-2} a_{n} P_{n}(\mu)\right]_{r=R+\Sigma a_{n} P_{n}} \\
=\frac{Q}{R^{2}}\left\{1+\sum_{n=1}^{\infty}(n-1) \frac{a_{n}}{R} P_{n}\right. \\
\left.-\sum_{n=1}^{\infty} \sum_{m=1}^{\infty}[(n+1)(n+2)-3] \frac{a_{n} a_{m}}{R^{2}} P_{n} P_{m}\right\}
\end{aligned}
$$

или

$$
\begin{aligned}
\sigma & \approx \frac{Q}{4 \pi R^{2}}\left\{1+\sum_{n=1}^{\infty}(n-1) \frac{a_{n}(t)}{R} P_{n}\right. \\
& \left.-\sum_{n=1}^{\infty} \sum_{m=1}^{\infty}[(n+1)(n+2)-3] \frac{a_{n}(t) a_{m}(t)}{R^{2}} P_{n}(\mu) P_{m}(\mu)\right\} .
\end{aligned}
$$




\section{Положение „эффективного“ заряда сореры}

Положение „эффективного“ заряда заряженной осциллирующей сферы определится выражениями

$$
\begin{gathered}
\mathbf{R}_{q}(t)=\frac{1}{Q} \int_{S} \mathbf{r} \sigma(\theta, t) d s, \\
S \equiv[r=r(\theta, t), \quad 0 \leq \theta \leq \pi, 0 \leq \varphi \leq 2 \pi], \\
\mathbf{r}=r\left(\mathbf{e}_{x} \sin \theta \cos \varphi+\mathbf{e}_{y} \sin \theta \sin \varphi+\mathbf{e}_{z} \cos \theta\right),
\end{gathered}
$$

где $\mathbf{e}_{x}, \mathbf{e}_{y}, \mathbf{e}_{z}$ - орты декартовой системы координат, не зависящие от положения точки пространства, в которой они определяются.

Наличие зависящего от времени центра „эффективного“ заряда капли превращает ее в излучатель электромагнитных волн дипольного типа. Выражение для интенсивности $I$ электромагнитного излучения от капли в соответствии с известным выражением [10] запишем в виде

$$
I=\frac{2}{3 c^{2}} Q^{2}\left(\frac{\partial^{2}}{\partial t^{2}} \mathbf{R}_{q}(t)\right)^{2} .
$$

Поскольку по предположению осцилляции поверхности капли обладают осевой симметрией, то смещения центра заряда капли в плоскости $x, y$ не происходит

$$
R_{q x}=R_{q y}=0 .
$$

В итоге запишем проекцию вектора смещения центра заряда вдоль оси $z$ в виде

$$
R_{q z}=\frac{1}{Q} \int_{S} \frac{\sigma(\theta, t)}{\mathbf{n}, \mathbf{e}_{r}} r^{3} \cos \theta \sin \theta d \theta d \varphi .
$$

Используя (3a) и (7), найдем $R_{q z}$ с точностью до $\left(\frac{a_{n}}{R}\right)^{2}$ :

$$
\begin{aligned}
R_{q z}^{*}= & \frac{Q}{4 \pi R^{2}} \frac{R^{3}}{Q} \int_{S}\left\{1+\sum_{n=1}^{\infty}(n-1) \frac{a_{n}}{R} P_{n}\right. \\
& \left.-\sum_{n=1}^{\infty} \sum_{m=1}^{\infty}[(n+1)(n+2)-3] \frac{a_{n} a_{m}}{R^{2}} P_{n} P_{m}\right\} \\
& \times\left[1+3 \sum_{n=1}^{\infty} \frac{a_{n}}{R} P_{n}(\cos \theta)+3 \sum_{n, m=1}^{\infty} \frac{a_{n} a_{m}}{R^{2}} P_{n} P_{m}\right] \\
& \times \cos \theta \sin \theta d \theta d \varphi \approx \\
\equiv & \frac{R}{2} \int_{-1}^{1}\left\{P_{1}+\sum_{n=1}^{\infty}(n+2) \frac{a_{n}}{R} P_{n} P_{1}\right. \\
& \left.-\sum_{n=1}^{\infty} \sum_{m=1}^{\infty}(n+1)(n+5) \frac{a_{n} a_{m}}{R^{2}} P_{n} P_{m} \mu\right\} d \mu .
\end{aligned}
$$

Несложно видеть, что, согласно (10), в линейных по $a_{n}(t) / R$ расчетах получается $R_{q z}=a_{1}$. Но в расчетах первого порядка нулевая и первая моды не возбуждаются в силу сохранения объема капли и неподвижности ее центра масс. Таким образом, в линейном приближении $a_{0}=0$ и $a_{1}=0$. Получается, что в предлагаемой модели в линейном приближении по амплитудам осцилляций интенсивность дипольного электромагнитного излучения равна нулю. Однако в линейных же расчетах [3-7] (полученных на основе анализа дисперсионного уравнения) получена интенсивность электромагнитного излучения, отличная от нуля. В [3-7] анализировалось дисперсионное уравнение для осциллирующей заряженной капли идеальной несжимаемой жидкости, и оказывалось, что частота осцилляций капли комплексна (а для идеальной несжимаемой жидкости она должна быть вещественной). Из этого обстоятельства делался справедливый вывод, что затраты энергии в подобном осцилляторе должны быть связаны с излучением электромагнитных волн. Исходя из этого интенсивность электромагнитного излучения в [3-7] оценивали по порядку величины.

Другими словами, излучение осциллирующих заряженных капель в линейных расчетах имеет место. Но поскольку дипольного излучения нет, то оно может быть квадрупольным. Квадрупольный момент осциллирующей сферической заряженной капли отличен от нуля (см., например, [17]). Собственно, в этой задаче рассчитывается квадрупольный момент заряженного проводящего сфероида. Но если рассмотреть осцилляции только основной моды сферической капли $(n=2)$, то это будут именно сфероидальные осцилляции, и по результату расчетов [17] можно оценить квадрупольный момент $D_{z z}$ сферической заряженной капли, совершающей сфероидальные осцилляции по порядку величины, усредняя вытянутую сфероидальную форму капли по полупериоду осцилляций. Квадрупольный момент будет порядка $\bar{D}_{z z} \sim Q\left(a^{2}-b^{2}\right)[17]$, где $a-$ большая и $b-$ меньшая полуоси вытянутой (осциллирующей) капли. Интенсивность квадрупольного излучения капли определится как [10]

$$
I=\frac{1}{180 c^{5}}\left(\frac{d^{3} D_{z z}}{d t^{3}}\right)^{2} \sim \frac{\omega^{6} \bar{D}_{z z}^{2}}{180 c^{5}},
$$

где $\omega-$ частота осцилляций капли, $c-$ скорость света. Учтем, что амплитуды сфероидальных осцилляций капель, наблюдаемые в реальных условиях, достигают половины радиуса $[18,19]$, и, принимая, что для капли воды с $R \sim 10 \mu \mathrm{m}$ заряд $Q$ будет $\sim 10^{-7} C G S E$ [20], $a \sim 1.1 R$, а также, что $\omega^{2} \sim \gamma / \rho R^{3}$, из (11) найдем $I \sim 10^{-44} \mathrm{erg} / \mathrm{s}$. Эта оценка по порядку величины совпадает с интенсивностью, полученной в [7] на основе анализа дисперсионного уравнения. Некоторое различие объясняется грубостью оценки данного рассмотрения, проведенной лишь по порядку величины.

Следует отметить, что зависимости интенсивности квадрупольного излучения от радиуса капли $R$ и от ее 
заряда $Q$, определяемые (11), полностью совпадают с таковыми в расчетах [3-7].

Перейдем к оценке интенсивности излучения во втором порядке малости по $a_{n} / R$. Учтем квадратичное по безразмерным амплитудам мод слагаемое в (10) $\left(\sim a_{n} a_{m} / R^{2}\right)$. В итоге получается

$$
\begin{aligned}
\left|R_{q z}\right| \approx & \frac{R}{2} \int_{-1}^{1}\left\{\sum_{n=1}^{\infty} \sum_{m=1}^{\infty}(n+1)(n+5) \frac{a_{n} a_{m}}{R^{2}} P_{n}(\mu) P_{m}(\mu) \mu\right\} d \mu \\
\equiv & \frac{R}{2} \sum_{n \in \Xi} \frac{2(n+1)(n+5)}{2 n+1} \\
& \times\left[\frac{a_{n} a_{n+1}}{R^{2}} \frac{(n+1)}{2 n+3}+\frac{a_{n} a_{n-1}}{R^{2}} \frac{(n-1)}{2} n-1\right] .
\end{aligned}
$$

Здесь $\Xi$ означает множество изначально возбужденных мод.

Далее, принимая, что временная зависимость амплитуд мод является гармонической: $a_{n} \sim \exp \left(i \omega_{n} t\right)$, получим

$$
\begin{aligned}
& \frac{\partial^{2} R_{q z}}{\partial t^{2}} \equiv \frac{\partial^{2}}{\partial t^{2}}\left\{\frac{R}{2} \sum_{n \in \Xi} \frac{2(n+1)(n+5)}{2 n+1}\right. \\
& \quad \times\left[\frac{\alpha_{n} \alpha_{n+1}}{R^{2}} \frac{(n+1)}{2 n+3} \exp \left[i\left(\omega_{n}+\omega_{n+1}\right) t\right]\right. \\
& \left.\left.\quad+\frac{\alpha_{n} \alpha_{n-1}}{R^{2}} \frac{(n-1)}{2 n-1} \exp \left[i\left(\omega_{n}+\omega_{n-1}\right) t\right]\right]\right\} \\
& \equiv\left\{\frac { R } { 2 } \sum _ { n \in \Xi } \frac { 2 ( n + 1 ) ( n + 5 ) } { 2 n + 1 } \left[\frac{\alpha_{n} \alpha_{n+1}}{R^{2}} \frac{(n+1)}{2 n+3}\right.\right. \\
& \quad \times\left(\omega_{n}+\omega_{n+1}\right)^{2} \exp \left[i\left(\omega_{n}+\omega_{n+1}\right) t\right] \\
& \left.\left.\quad+\frac{\alpha_{n} \alpha_{n-1}}{R^{2}} \frac{(n-1)}{2 n-1}\left(\omega_{n}+\omega_{n-1}\right)^{2} \exp \left[i\left(\omega_{n}+\omega_{n-1}\right) t\right]\right]\right\},
\end{aligned}
$$

где $\alpha_{n}-$ не зависящие от времени максимальные значения амплитуд мод.

Для оценки по порядку величины в полученном выражении экспоненты с мнимым показателем заменим единицей.

В итого интенсивность электромагнитного излучения по (9) примет вид

$$
\begin{aligned}
I & =\frac{2}{3 c^{3}} Q^{2}\left(\frac{\partial^{2}}{\partial t^{2}} \mathbf{R}_{q z}\right)^{2} \equiv \frac{2 Q^{2}}{3 c^{3}} \\
& \times\left\{\frac { R } { 2 } \sum _ { n \in \Xi } \frac { 2 ( n + 1 ) ( n + 5 ) } { 2 n + 1 } \left[\frac{\alpha_{n} \alpha_{n+1}}{R^{2}} \frac{(n+1)}{2 n+3}\left(\omega_{n}+\omega_{n+1}\right)^{2}\right.\right. \\
& \left.\left.+\frac{\alpha_{n} \alpha_{n-1}}{R^{2}} \frac{(n-1)}{2 n-1}\left(\omega_{n}+\omega_{n-1}\right)^{2}\right]\right\}^{2}
\end{aligned}
$$

Для $n=2, \alpha_{n} / R \sim 0.1$, в предположении, что амплитуды всех возбужденных мод одинаковы, оценка интенсивно- сти излучения по (12) совпадает по порядку величины с результатом нелинейных расчетов [9], а именно $I \sim 10^{-30} \mathrm{erg} / \mathrm{s}$.

Зависимости интенсивности дипольного излучения от радиуса капли $R$ и заряда $Q$, определяемые (12), полностью совпадают с таковыми в расчетах [9].

Согласно общей теории электромагнитного излучения [11], отношение интенсивности квадрупольного излучения к интенсивности дипольного по порядку величины равно отношению гидродинамической скорости движения к скорости света в квадрате: $V^{2} / c^{2} \approx R^{2} \omega^{2} / c^{2}$, что при принятых значениях физических величин составляет $\sim 10^{-14}$, как и получено в проведенных оценках.

\section{Заключение}

Предложена математическая модель дипольного и квадрупольного излучений, позволяющая рассчитать его интенсивность как в линейных, так и в нелинейных расчетах. В расчетах первого приближения по амплитуде осцилляций дипольного излучения электромагнитных волн от заряженной капли не обнаруживается. Получаемое в этих расчетах излучение определяется квадрупольной компонентой полного излучения. Квадрупольная компонента оказывается на много порядков величины менее интенсивной, чем дипольная. Дипольное излучение обнаруживается лишь в расчетах более высоких порядков малости по амплитуде осцилляций, чем квадрупольная, однако его интенсивноть много больше (на четырнадцать-пятнадцать порядков величины) интенсивности квадрупольного излучения. В естественных условиях электромагнитное излучение состоит из трех компонент: дипольного, квадрупольного и магнтнодипольного (того же порядка, что и дипольное, но часто отсутствующее). Когда есть дипольное излучение, квадрупольное на его фоне не заметно. Когда нет дипольного излучения, самое интенсивное излучение квадрупольное. Именно его и предсказали в первой работе на эту тему [3].

Работа выполнена при поддержке грантов РФФИ № 14-01-00170-а и 14-08-00240-а.

\section{Список литературы}

[1] Качурин Л.Г. Физические основы воздействия на атмосферные процессы. Л.: Гидрометеоиздат, 1990. 463 с.

[2] Белоцерковский А.В., Дивинский Л.И. и др. Активнопассивная радиолокация грозовых и грозоопасных очагов в облаках / Под ред. Л.Г. Качурина, Л.И. Дивинского. СПб: Гидрометеоиздат, 1992. $286 \mathrm{c.}$

[3] Калечии, В.И., Нахутин И.Е., Поуэктов П.П. // ДАН CCCР. 1982 T. 262. C. 1344-1347.

[4] Богатов Н.А. Электромагнитное поле, генерируемое капиллярными колебаниями капель // Сборник тезисов докладов VI Международной конференции „Солнечноземные связи и физика предвестников землетрясений“. Петропавловск-Камчатский, ДВО РАН. 2013. С. 22-26. 
[5] Григорьев А.И., Ширяева С.О., Головано А.С., Рыбакова М.В. // ЖТФ. 2002. Т. 72. Вып. 1. С. 8-14.

[6] Григорьев А.И., Ширяева С.О. // Известия РАН. МЖГ. 2002. № 5. C. 67-73.

[7] Grigoryev A.I., Kolbneva N.Yu., Shiryaeva S.O. // Surf. Eng. Appl. Electrochem. 20015. Vol. 51. N 6. P. 530-539.

[8] Ширяева С.О. // ЖТФ. 2002. Т. 72. Вып. 4. С. 15-19.

[9] Ширяева С.О., Григорьев А.И., Колбнева Н.Ю. // ЖТФ. 2016. Т. 86. Вып. 3. С. 41-50.

[10] Ландау Л.Д., Лифиии, Е.М. Теория поля. М.: Наука, 1973. $504 \mathrm{c}$.

[11] Савельев И.В. Основы теоретической физики. Т. 1. М.: Наука, 1975. $416 \mathrm{c}$.

[12] Френкель Я.И. // ЖЭТФ. 1936. Т. 6. № 4. С. 348-350.

[13] Варшалович Д.А., Москалев А.Н., Херсонский В.К. Квантовая теория углового момента. Л.: Наука, 1975. 436 с.

[14] Hendrics C.D., Schneider J.M. // J. Amer. Phys. 1963. Vol. 1. N 6. P. $450-453$.

[15] Rayleigh (Strutt J.W.) // Phil Mag. 1882. Vol. 14. P. 184-186.

[16] Ландау Л.Д., Лифиии, Е.М. Гидродинамика. М.: Наука, 1986. $733 \mathrm{c}$.

[17] Ландау Л.Д., Лифиии Е.М. Электродинамика сплошных сред. М.: Наука, 1957. 532 с.

[18] Beard K.V., Tokay Ali. // Geophys. Res. Lett. 1991. Vol. 18. N 12. P. 2257-2260.

[19] Стерлядкин В.В. // Известия АН СССР. Физика атмосферы и океана. 1988. Т. 24. N 6. С. 613-621.

[20] Мазин И.П., Хргиан А.Х., Имянитов И.М. Облака и облачная атмосфера. Справочник. Л.: Гидрометеоиздат, 1989. $647 \mathrm{c}$. 